\title{
Memoria indígena y dictadura en contextos de transición: caso Aikewara"
}

Andrea Ponce García**

RESUMEN

CON EL PROPÓSITO DE REFLEXIONAR SOBRE LA MULTIDIMENSIONALIDAD DE LA MEMORIA Y SUS MULTIFACÉTICAS EXPResiones, este artículo eXPone el Caso particular del pueblo indígena Suruí AikeWara, aCtualmente ubicado en la región sudeste del estado de Pará en Brasil.

DESPÚES DE QUE LA DICTADURA CIVIL MILITAR LLEGARA A SU FIN EN 1985 SE ASUMIÓ QUE EXISTÍA UN SILENCIO COLECTIVO

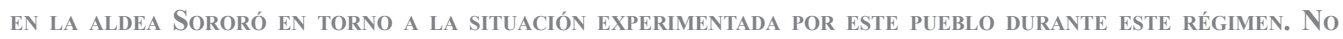
OBSTANTE, VARIOS FACTORES de LA PROPIA CONSTRUCCIÓN DE POLÍTICAS DE LA MEMORIA Y DE LA LLAMADA 'JUSTICIA TRANSICIONAL' BRASILEÑA HAN INCIDIDO -ASÍ COMO OTRAS MEDIACIONES- PARA QUE EL REPERTORIO NEMÓNICO AIKEWARA HAYA SIDO COLOCADO EN ESPACIOS CADA VEZ MÁS PÚBLICOS A NIVEL NACIONAL.

Durante el proceso de instalación y (Des) instalación del ETHOS del Silencio (ARruti, 2006) SE Fue CONFIGURANDO UNA RED SOCIAL CON LA CUAL ESTE GRUPO COMENZÓ A INTERACTUAR CON EL PROPÓSITO DE QUE SU VERSIÓN DE LA HISTORIA PASARA TAMBIÉN A FORMAR PARTE DE LA 'CONTROVERSIA PÚBLICA' Y REINVINDICAR EL IMAGINARIO CREADO EN TORNO A ELLOS Y SU SUPUESTA PARTICIPACIÓN EN CAMPAÑAS MILITARES "ANTI TERRORISTAS". De tal Suerte, el presente artículo subyacentemente Se proyecta a tensar un poco más los posibles HILOS ENTRE CONCEPCIONES NATIVAS DE JUSTICIA, MEMORIA Y LOS PROPIOS DERECHOS DE AMNISTÍA Y REPARACIÓN EN CONJUNCIÓN CON PARTICULARIDADES Y CONTEXTOS INDÍGENAS.

Palabras Clave: Suruí Aikewara - memoria - dictadura - territorio - violencia.

Abstract

IN ORDER TO REFLECT ON THE MULTIDIMENSIONALITY OF MEMORY AND ITS MULTIFACETED EXPRESSIONS, THIS ARTICLE presents the case of the Surui tribe Aikewara, currently located in the southeast of Para state in Brazil. After the CIVIL-MiLITARY DiCTATORSHIP CAME TO AN END IN 1985 IT WAS ASSUMED THAT THERE WAS A COLLECTIVE SILENCE IN THE Sororo VILLAge ABOUT THE SITUATION EXPERIENCED BY THE PEOPLE DURING THIS REGIME. HoWEVER, SEVERAL FACTORS REGARDING THE CONSTRUCTION OF MEMORY POLITICS AND THE SO-CALLED 'TRANSITIONAL JUSTICE'

* El presente artículo corresponde a una síntesis de una parte de la disertación (principalmente de los dos primeros capítulos) titulada: "Trayectoria de la(s) memoria(s) Aikewara: del evento de la Guerrilla de Araguaia a la Comisión de Amnistía en el actual contexto de revisión de la dictadura brasileña", la misma que fue defendida por la autora en 2015 en la Universidad Estatal de Campinas- UNICAMP, Brasil.

** Master en Antropología Social por la UNICAMP. Su licenciatura fue realizada en la PUCE dentro de la escuela de Antropología. Los principales ejes de interés de la autora giran en torno a la antropología política, movimientos sociales, movildad humana y la ecología política. Actualmente se encuentra trabajando como oficial de capacitación con organizaciones campesinas en la Fundación Heifer Ecuador. Correo electrónico: andrea_ponceg@yahoo.com 
HAVE AFFECTED OF BRAZIL -AS WELL AS OTHER MEDIATIONS HAVE RESULTED IN AIKEWARAN MNEMONIC REPERTOIRE BEING INCREASINGLY USED IN PUBLIC SPACES NATIONWIDE.

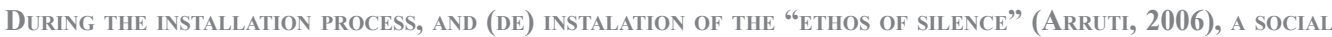
NETWORK AROSE WHICH BEGAN TO INTERACT WITH THE PURPOSE OF ITS VERSION OF THE STORY BECOMING PART OF THE 'PUBLIC CONTROVERSY' AND RE-VINDICATING THE IMAGE CREATED AROUND THEM AND THEIR ALLEGED INVOLVEMENT IN MILITARY "ANTI TERRORIST" CAMPAIGNS.

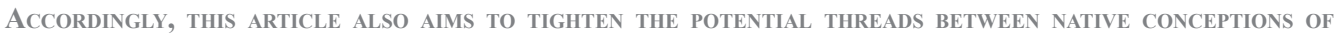
JUSTICE, MEMORY AND THE RIGHTS OF AMNESTY AND REPARATIONS IN CONJUNCTION WITH INDIGENOUS PARTICULARITIES AND CONTEXTS.

KeYwords: Surubí AiKewara - MEMORY - DiCTATORSHiP - TERRITORY - VIOLENCE.

\section{Introducción}

Antes de llegar a Brasil para realizar mis estudios cargaba ya un acumulado interés especial en relación a las particularidades de las dictaduras militares sentidas en las distintas latitudes de América Latina. La oportunidad se me había presentado: el orientador de tesis me propuso trabajar alrededor de pueblos indígenas y dictadura militar brasileña; sin dudarlo me embarqué en un trayecto que me impresionó de varias maneras de principio a fin.

Sobre el caso del período dictatorial brasileño (1964-1985), la mayoría de datos se han centrado en develar la realidad sentida por las víctimas ${ }^{1}$ registradas en varios centros urbanos del país, sin embargo fue con la creación e instalación de la Comisión de la verdad ${ }^{2}$ nacional (la última en América Latina) que la situación experimentada por los diversos grupos indígenas comenzó a ser tímidamente reflexionada y socializada públicamente.

Como digo, en base a los primeros levantamientos de información me deparé con casos de genocidios programados como el de los Waimiri-Atroari ${ }^{3}$ (por citar un ejemplo entre muchos otros), con relatos de prisiones y reformatorios creados para reclutar indígenas "problemáticos" de varias etnias, -siendo el caso más conocido el del Reformatorio Krenak ${ }^{4}$ - .El panorama se complejizaba más aún con el reconocimiento de la existencia de programas del ejército brasileño direccionados a armamentizar y militarizar a un grupo de indígenas en la 'Guarda rural indígena'.

Contando ya con varios elementos de análisis, sucedió un día en el que conversando con la persona ${ }^{5}$ que justamente había sido la responsable de sacar a luz al llamado 'Relatorio

1 En base a los recientes resultados publicados en el tercer volumen del Informe final de la Comisión Nacional de la Verdad-Brasil (diciembre 2014) se contabiliza un total de 210 desaparecidos, 191 muertos y 33 cuerpos localizados, dando un estimativo total de 434 muertos y desaparecidos durante la dictadura civil militar en Brasil, contabilizando 377 responsables de tales crímenes (Informe CNV, 2014). Ver también http://g1.globo.com/politica/noticia/2014/12/ consulte-integra-do-relatorio-final-da-comissao-nacional-da-verdade.html.

2 La Comisión Nacional de la Verdad (CNV) en Brasil fue creada por Ley 12528/2011 e instalada el 16 de mayo de 2012 con el fin de investigar las graves violaciones de los derechos humanos que tuvieron lugar en el país durante el período de septiembre de 1946 hasta octubre de 1988 (siendo los dos años en los que se reconocieron nuevas constituciones democráticas) y se conformó por varios grupos de trabajo (GT), uno de ellos relacionado directamente a campesinos y pueblos indígenas. Durante el tiempo en el que se llevaba a cabo el estudio la representante de este GT era la psicoanlista María Rita Kelh.

3 Ver Baines, 1991 e 1 Relatório do Comitê Estadual da Verdade 'O genocidio do povo Waimiri Atroari', 2012.

4 Entre las estrategias a las cuales el régimen militar recurrió para desplazar grupos indígenas estuvo la creación del 'Reformatorio agrícola indígena Krenak', centro al cual serían enviados indígenas catalogados de 'problemáticos' o 'peligrosos', funcionando así como una verdadera cárcel indígena a nivel nacional. De igual manera, en ese mismo contexto, vale la pena mencionar la existencia de la llamada Guarda Rural Indígena (GRIN), cuya primera turma sería constituida en 1969 por cerca de 84 indígenas provenientes de un variado mosaico étnico bajo coodinación del Capitán de la Policia de Minas Gerais, Manuel Santos Pinheiro (Freitas, 2011: 2).

5 Marcelo Zelic es vicepresidente del Grupo Tortura Nunca Mais, SP. Y fue quien recuperó al Informe Figueiredo en el Museo del Indio, RJ, después de que durante años las autoridades habían señalado que el documento se habría perdido en un incendio. 
Figueired ${ }^{6}$, pude conocer sobre la situación particular de un pueblo indígena que sería víctima directa de las acciones militares.

En Brasil en plena dictadura civil militar surgieron algunos movimientos de resistencia y oposición al régimen7, siendo que la 'Guerrilla de Araguaia' pasó a ser de los más reconocidos. Miembros del ex $\mathrm{PCdoB}^{8}$ se habían instalado en la región conocida como 'Bico do Papagaio' al sudeste del estado de Pará' con el objetivo de combatir al ejército de forma armada.

Desde la instalación de los primeros guerrilleros en la zona (1964) hasta su aniquilación (1975) liderada por el ejército nacional, el pueblo Suruí Aikewara se vio inmerso en un 'fuego cruzado' que como veremos le representó una serie de violaciones sistemáticas que se extienden hasta hoy en día. Como una suerte de recordatorio constante de lo sucedido está la autopista BR 153 (anterior OP2), construída por las tropas militares para facilitar su ingreso a las zonas de posible incursión guerrillera y que terminó desmembrando al territorio indígena.

Por tal motivo, el objetivo central que se perseguió durante el desarrollo de esta investigación fue el de etnografiar el proceso de revitalización de las memorias especializadas y colectivas de este pueblo indígena particular sobre un evento ${ }^{10}$ que los ha marcado generacionalmente, problematizando los silencios y más recientemente, las distintas maneras de narrarlo, en contextos donde los Aikewara se han visto inmersos en sí en negociaciones de códigos sobre

6 El Informe Figueiredo presentado en 1988 es el resultado de una Comisión Parlamentar de Inquérito - CPI de Investigación del Ministerio del Interior que fue presidida por el procurador federal Jader de Figueiredo Correia, creada para verificar la situación de los indígenas en Brasil. De cierta manera, a causa del contenido revelado sobre las faces de violencia física y simbólica, genocídio, epidemias, acciones disciplinares de integración practicados contra diversos pueblos indígenas en sus puestos, el Informe Figueiredo atribuyó al Servicio de Protección a los Indios (SPI) la responsabilidad de gran parte del sufrimiento e incluso del extermínio, concluyendo que 80 pueblos indígenas habían desaparecido por completo.

Como consecuencia el extinto SPI pasó a convertirse en la Fundación Nacional del Indio (FUNAI), "com incumbência de construir um novo modelo de indigenismo no Brasil baseado na segurança e desenvolvimento, porém, assim como o SPI, a FUNAI esteve pautada na necessidade de integração do índio à sociedade nacional e fez prevalecer o estímulo à mudança (aculturação)" (file:///C:/Users/Martha\%20Pacheco/Downloads/relatoriofigueiredo-ditadura-militar-e-torturas-indigenas.pdf).

7 Entre los que se puede citar a: la Vanguarda Popular Revolucionaria (VPR), Organização Política MarxistaLeninista Politica Operária (Polop), Vanguarda Armada Revolucionária-Palmares (VAR-Palmares), Movimento Revolucionário 8 de Outubro (MR-8), Partido Comunista Brasileiro Revolucionário (PCBR), Ação Libertadora Nacional (ALN) y Comandos de Libertação Nacional (COLINAS) (Gorender, 1987).

8 Los partidos de oposición al gobierno civil militar instaurado se vieron inmersos en procesos de discrepancias internas, surgiendo procesos de división política ideológica que dieron vida a nuevas corrientes políticas revolucionarias disidentes del Partido Comunista Brasileiro (PCB). Lo que estaba en discusión era si las nuevas propuestas políticas seguirían modelos chinos, soviéticos o de inspiración cubana (Campos Filho, 2003): la divergencia entre los partidos de izquierda se concentraba en el tipo de resistencia a ser asumida. En ese contecto surgiría el llamadol Partido Comunista do Brasil (PCdoB).

Entre las organizaciones de izquierda se dio una diferenciación entre aquellas que se basaban en el foquismo y que defendían una lucha armada inmediata, en contraposición, a otra fracción que se focalizaba en la lucha popular prolongada, de inspiración maoísta. Siendo éste el caso del grupo liderado por João Amazonas, Maurício Grabois, Ângelo Arroyo y Pedro Pomar en el Partido Comunista do Brasil (PCdoB), cuya 'reorganización' se remonta a 1962 después de la fragmentación del PCB, creado en 1922 (Gorender, 1987; Fernandes, 2013).

9 El territorio del estado paraense, al encontrarse dentro de la denominada jurisdicción de la 'Amazonía legal' desde 1966, no han sido pocos los conflictos generados en torno al control de los recursos estratégicos de interés nacional e internacional. Para citar el caso más conocido: en 1980 tuvo inicio la mega extracción de oro a cielo abierto en 'Serra Pelada', mina localizada en el actual municipio de Curionópolis, nombre en alusión al Mayor 'Curió' (o Dr. Luchini durante época de represión a la Guerrilla de Araguaia), quien aún hoy se consolida como "uma onipresença nesse meio..." (Nossa, 2012: 483).

10 Se debe recalcar que a lo largo de este texto recurrimos a la propuesta teórica-conceptual de evento crítico (Das: 1995, 2007) para reflexionar sobre lo que sería la represión a la guerrilla desde la perspectiva Aikewara. Conforme la lectura analítica de un evento pasa categorizar situaciones que a causa de sus importantes implicancias sociales forjan un 'antes' y un 'después' en la cotidianidad de las personas y que da paso a la narrativa como vehículo mediante el cual, estos eventos críticos se extienden y pasan a ser re-constituidos en las relaciones sociales presentes en base al hecho pasado.

La propuesta teórica de Das $(1995,2007)$ a partir de la cual se dará lectura a los eventos analizados, es absolutamente pertinente en cuanto nos alerta sobre la interacción que cada uno de ellos tiene con la cotidianidad de los sujetos que los vivenciaron ellos o mediante las narrativas de los otros. Siendo que con tal herramienta se puede ir construyendo nuevas propuestas de etnografar la violencia. 
verdad, justicia y reparación, formando parte de procesos llevados adelante por la Comisión Nacional de Verdad (CNV) y la Comisión de Amnistía (CA).

La presencia y agencia de este pueblo indígena en el evento de Araguaia los ha colocado historiográficamente en un lugar inestable, donde a veces son considerados las víctimas y en otras ocasiones, los victimarios, y por ello su "papel" en este episodio particular de la historia nacional está siempre en constante negociación.

Esta investigación me permitió entre varios otros asuntos, reflexionar sobre la posibilidad de cuestionarnos acerca de la paradoja entre el deseo del silenciamiento de tal evento y la emergencia del reconocimiento de los Suruí Aikewara como actores-productores de nuevas versiones sobre ellos mismos; de preguntarnos ¿qué es lo que conecta la "memoria controlada" en un tiempo pasado, con la reciente producción de procesos que los movilizan a re-contar los hechos?

En esa misma línea, diferentes perspectivas pueden ser observadas para leer ese evento como marco que desplaza a los Aikewara de su supuesta posición de 'colaboradores' de los militares en plena dictadura, re-colocándolos ahora, como víctimas, y en extensión como sujetos de derechos de reparación.

\section{Los nuevos encuentros Aikewara}

Los Aikewara $^{11}$ (tronco Tupi) de la aldea Sororó ${ }^{12}$ han atravesado una serie de transformaciones inscritas en las fugas que han emprendido a partir de ataques inter-tribales y, posteriormente, a raíz de los primeros contactos con los "blancos" (en lengua nativa ${ }^{13}$ : kamará).

La primera tentativa de pacificación de los Aikewara fue realizada en 1951 por el fray dominicano Gil Gomes Leitão, sin embargo, fue en 1953, cuando el religioso logró concretizar el encuentro con cerca de cien indígenas localizados junto a un igarapé (Ferraz, 2013). Este primer contacto fue reforzado con más expediciones de la misma orden religiosa durante el periodo comprendido entre 1954-1958.

En 1947 y 1957 fueron recibidos a bala por castañeros ${ }^{14}$ locales o fueron cooptados por regionales influyentes para cazar pieles y, en la década de los sesenta sufrieron una importante reducción poblacional a causa de una epidemia de gripe, originada post contacto. Razón por la cual existen hipótesis que se refieren a la ejecución de arreglos poliándricos ${ }^{15}$ al interior de la aldea Aikewara para lograr contrarestar su cuasi extinción.

11 A lo largo de este trabajo se ha optado por utilizar la denominación completa Suruí Aikewara o solamente Aikewara, al haber sido ésta la sugerencia proveniente de gran parte de los pobladores actuales de la aldea Sororó, quienes me refirieron que 'Suruí' sería la denominación más 'estatal', mientras que su forma de auto-identificarse es 'Aikewara' (gente). Diario de campo, abril, 2014.

12 Cabe señalarse que desde 2003, los Aikewara se encuentran divididos en dos aldeas: Sororó, localizada a $2 \mathrm{~km}$ de la autopista BR-153, con 315 individuos; e Itahy, ubicada junto al río Gameleira, en el límite sudeste, a cerca de 1,5 $\mathrm{km}$ de la autopista con aproximadamente 40 personas.

Itahy surgió como resultado de una separación de familias y en donde actualmente vive la descendencia de un ex cacique y él mismo, quienes en su mayoría se reconocen como evangélicos. Pese a que durante mi trabajo investigativo no me fue posible develar a profundidad los impactos de tal fragmentación, para el tema abordado en este artículo al menos es importante tomar en cuenta que es recurrente el uso de dispositivos discursivos que buscan categorizar y legitimar a un solo grupo como 'verdaderamente indígena' (refiriéndose a los que viven en Sororó) en contraposición a quienes viven en la aldea reciente.

Así mismo se debe reseñar que varios actores sociales que de una u otra manera forman parte de la problemática aquí analizada, han evocado que en Itahy se vislumbra una nueva ‘élite política' que tiene mayor incidencia en el plano de la ‘controversia pública' (Arruti, Montero, Pomba, 2012).

13 Se trata de una etnia clasificada junto al tronco lingüístico Tupi, que junto a otros catorce pueblos se localiza en la región sudeste del estado de Pará, Brasil. De acuerdo con Rodrigues (1986), los Aikewara hablan la lengua akwáwa, que pertenece al subconjunto IV de la familia Tupi-Guaraní; no obstante, actualmente gran parte de la población habla también portugués.

14 El área es reconocida por la presencia de varios árboles de las castañas-do-pará, las mismas que resultan un producto estratégico para la producción. Actualmente la mayoría de Aikewara vende sus castañas cosechadas a intermediarios que van hasta la aldea Sororó para adquirir el producto.

15 Entrevista Prof. Roque Laraia, 14 de mayo, 2014. UnB, Brasilia. 
Sumando a ello, desde 1964 aparecen a escena unos nuevos actores con los cuales los Aikewara mantendrían diferentes niveles de interacción y relacionamiento.

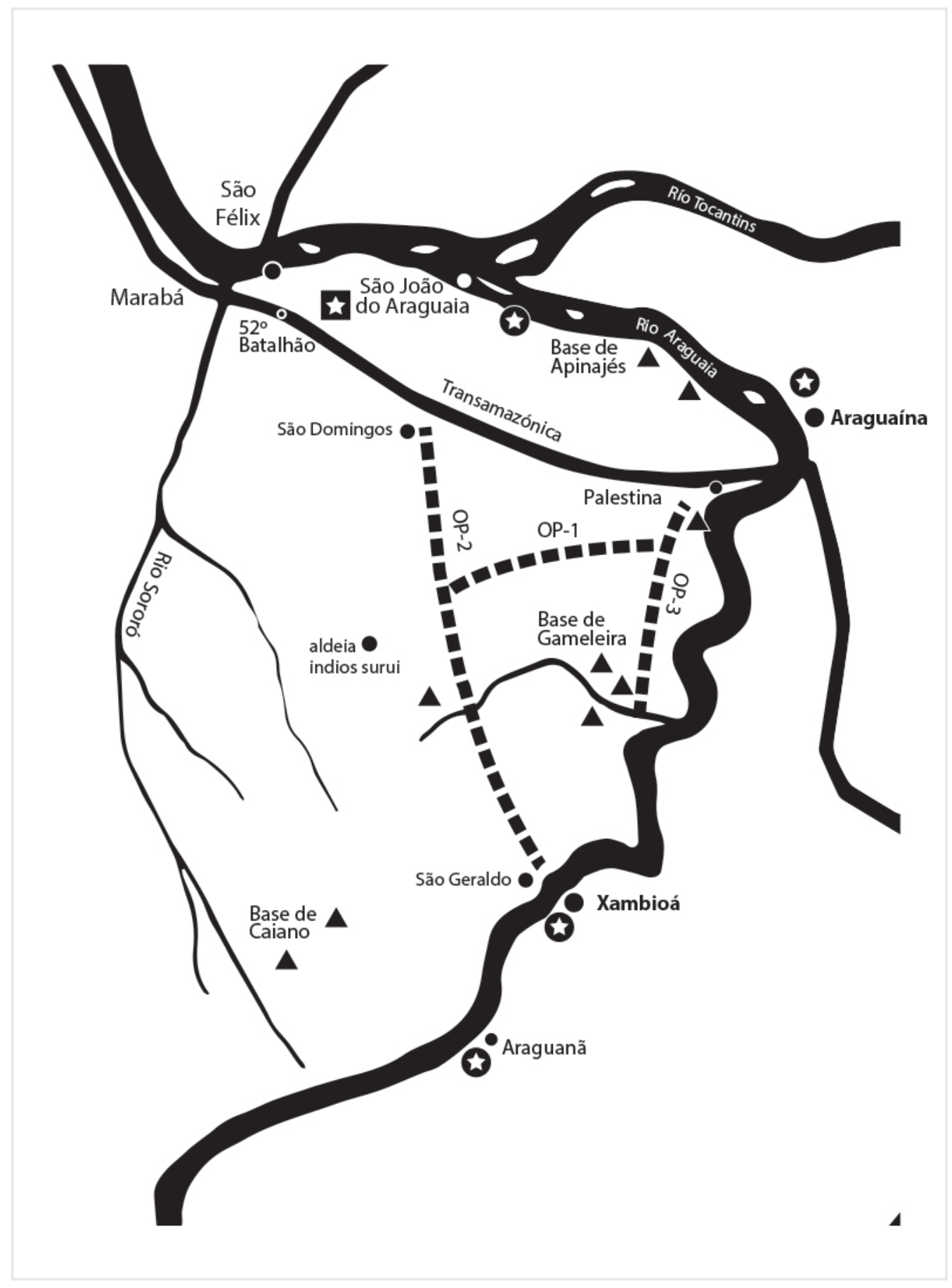




\section{El encuentro con los 'paulistas ${ }^{16}{ }^{\text {'- 'terroristas }}{ }^{17}$ '- 'desaparecidos políticos'}

Osvaldo O. da Costa (Osvaldão) fue el primer miembro del PCdoB a localizarse en la región desde 1966, y ya el 25 de diciembre de 1967 desembarcarían: Mauricio Grabois (Velho Mário), Elza Monnerat (Dona Maria) y el médico João Carlos Haas (Juca), quien ejercería la función de jefe de salud de la guerrilla, uniéndose después Gilberto Olimpio (Pedro Gil), entre otros. Según Peixoto (2011), ya para 1968 eran 15 personas las que conformaban el movimiento guerrillero, creciendo a 70 militantes aproximadamente en 1972.

Vale recalcar que el movimiento guerrillero promovió la "concientización política" entre los pobladores, creándose la União pela Liberdade e pelos Direitos do Povo (ULDP), que de una u otra manera terminaría supliendo algunos de los vacíos dejados por el Estado en temas de salud y eduacción, principalmente. Se sabe que inclusive relaciones de compadrazgo fueron forjadas en este encuentro entre campesinos de la región y los foráneos.

Con la mayoría de Aikewara y campesinos locales con los que pude conversar u acceder a los registros de sus relatos ${ }^{18}$ parecía existir una cierta constante en relación a la apreciación de los foráneos que iban ubicándose en la región paulatinamente, la mayoría de ellos conserva de alguna manera una imagen positiva de ellos. Pese a que contactos más directos se conocen entre campesinos y miembros de las Fuerzas armadas de Araguaia (ver Fernandez, 2013), entre los Aikewara existe incluso el caso de uno de ellos que fue práctivamente adoptado por el grupo de 'paulistas' durante un período.

Por otro lado, se podría decir que los idearios de la guerrilla en Araguaia no vislumbraban a los indígenas como posibles miembros de la organización, y es dable también apuntar que no parece haber existido una preocupación por parte del partido de establecerse en lugares cercanos a una tierra indígena.

De todas formas aún hoy cuando les preguntaba'19 acerca de los 'paulistas' la mayoría de personas en la aldea Sororó con quienes conversé, no negaban haber oído al menos de ellos, sin embargo era generalizada la respuesta en la que enfatizaban que desconocían quienes eran y cuales sus objetivos en la región.

\section{Al encuentro con los militares o ' ${ }^{\prime} \operatorname{arehai}^{20}$ '}

Cuando las noticias de la existencia de núcleos subversivos y terroristas llegaron a los altos mandos militares, se dio inicio a las operaciones militares en la región de 'Bico do papagaio'.

16 Paulistas era la manera generalizada por la cual los militantes del PCdoB eran designados en la región. La categoría colocada daba cuenta de una diferenciación percibida entre los pobladores y aquellos migrantes que habían ido poblando la región en base a una colonización espontánea o dirigida (Hébette, 2004).

17 Término mediante el cual los militares llamaban a los guerrilleros.

18 Ver http://www.funai.gov.br/index.php/comunicacao/galeria-de-videoss/3061?view=pgvideo\&institucionais limitstart=0\&sugeridos_limitstart=0\#galeria, vídeos funai http://www.ihu.unisinos.br/noticias/535443-indigenasaikewara-na-comissao-da-anistia-perdao-e-reparacao.

19 La segunda fase fue direccionada a la realización del trabajo de campo. La opción metodológica escogida para aproximarme a la multiplicidad de escenarios, en los que se desenvuelve esta problemática fue la de llevar a cabo una etnografía nómada (Arruti, 2006). De tal forma, conviví en la aldea pero también me desplacé junto con algunos Aikewara hacia los diferentes espacios en donde transitaban para socializar su 'verdad' y en búsqueda de que sus derechos indemnizatorios fueran reconocidos y agilizados.

Esta incluyó primeramente un tiempo de permanencia en la ciudad de Belém y Marabá (febrero- abril 2014) y en la aldea Sororó (26 de abril - 03 de junio 2014). Posteriormente, la trayectoria analizada incluyó la sesión llevada a cabo en Brasilia en la CNV, y meses después en el Ministerio de Justicia (Comisión de Amnistía).

Cada uno de estos eventos públicos fue considerado también como un escenario privilegiado para analizar etnográficamente el trabajo de encuadramiento de la memoria contando con soportes y mediadores -internos o externos- responsables del control de la imagen del grupo y de la dosis de objetividad/subjetividad en el proceso de reconstrucción y rememoración de los hechos narrados.

20 Traduciendo literalmente resultaría: no indio - militar. En lengua nativa, la traducción que los Aikewara han dado para la palabra portuguesa 'militar' o 'soldado' es la de marehai. Desde una aproximación más etnológica, los marehai serían los guerreros que no devoran a sus víctimas, que viven para-la-guerra; al contrario de los matadores (punurusukawae). El Marehai flecha a su presa y parte, y después entra en un proceso de purificación que le garantice expulsar, vomitar a su duplo" (Calheiros, 2014: 232). 
Con "Manobrão" (abril de 1972 - Junio 1972) ocurrió la primera investida militar en la zona. El ataque militar en un local denominado 'Pau Preto' ocasionó la movilización de los guerrilleros a las profundidades de la selva, mientras se iba convirtiendo a Marabá ${ }^{21}$ y Xambioá en ciudadescuarteles.

Para conseguir obtener la simpatía local y facilitar la búsqueda, el Ejército pasó a desarrollar la llamada 'Operación Aciso' (Ação Cívico- Social), llevando para esa región históricamente olvidada servicios asistencialistas como: médicos, vacunas, remedios. La Aciso se vio inmersa en conflictos por la tierra. Incluso, varios pobladores -aunque recelosos- se "beneficiaron" de estos servicios, aunque ello "naturalmente serviu para que a população fosse debidamente cadastrada" (Íbid, 2012: 154).

Fue durante la segunda campaña que el Ejército se valió de la participación forzada de algunos moradores y de los indígenas Aikewara para actuar como guías en la selva en la caza de los militantes.

En una tercera campaña se llevó a cabo la verdadera caza de guerrilleros, valiéndose de acciones de inteligencia entre la población civil. Se realizaron decapitaciones y corte de manos para identificación de guerrilleros asesinados y el Ejército además se esmeró en dejar un temor instaurado entre la población, reforzando en el imaginario local la idea de que con los últimos guerrilleros asesinados, cualquier posibilidad de subversión moriría junto con ellos. Campesinos de la región y algunos Aikewara no olvidan el día (durante el mes de abril de 1974) en el que Osvaldão fue colgado de un helicóptero y fue expuesto muerto desde las alturas. El mensaje de intimidación había sido enviado.

Se conoce que de los 98 guerrilleros: 41 de ellos fueron fusilados y otros 18, muertos en combates durante las tres campañas emprendidas, resultando así un número aproximado de 39 desaparecidos. De parte de las Fuerzas estatales hay registro de 6 agentes muertos y 1 desaparecido (Nossa, 2012: 16).

\section{Las tres memorias}

La memoria aquí está siendo entendida como un género de trabajo colectivo asociado a una determinada categoría de personas que se desenvuelven socialmente en forma de una suerte de 'convencionalización' (Bossi, 2006) que modela a los recuerdos de una manera particular y culturalmente coherente en un contexto de ideas y valores específicas que actúan sobre los hechos del pasado, modelaje que implica así mismo viabilizar un universo discursivo propio. Los recuerdos son colectivos en cuanto son evocados por otros, tanto en momentos de recordación o de la propia experiencia, siendo que las sucesivas evocaciones que la sociedad (en cuanto asociación de intereses) trae consigo son las que contribuyen en la construcción de la experiencia.

En el caso concreto de los Aikewara, abordaremos en total tres tipos de memorias: las especializadas, la común y la re-actualizada. Acerca del primer tipo se puede agregar que retratan experiencias diferentes, más complementarias en el sentido de que la rememoración personal se sitúa en donde se entrecruzan redes múltiples en las que estamos implicados, en el encuentro de una semántica con una pragmática (Ricoeur, 2008).

Recuerdos generados entre los que fueron y los que quedaron en la aldea durante los momentos de represión a la Guerrilla de Araguaia más que definir la experiencia fenomenológicamente, nos permiten dar cuenta de relaciones diferenciales. La sucesión de recuerdos -incluso aquellos más personales- puede ser explicada por los cambios que se producen en nuestras relaciones con los distintos medios colectivos y por las propias transformaciones de estos medios (Halbwachs, 2004).

21 Hasta el día de hoy Marabá es conocida regionalmente también como "Marabala" dado el elevado índice de violencia que presenta con acciones registradas de pistoleros pagados que actua(ban) a favor de grandes emprendimientos y velando por la permanencia de latifundios. 


\section{Memorias especializadas}

\section{a). Quienes partieron}

Siendo tan sólo cerca de cuarenta personas en la aldea, en 1972, se suscitó un evento: los Aikewara vieron su territorio invadido de militares (kamará marehai), quienes llegarían anunciando su lucha contra los militantes del PCdoB, que se habían instalado en la región desde inicios de los años sesenta bajo insignias de lucha armada y apoyo popular rural. Evento que dentro de la historiografía brasileña es conocido como la represión a la guerrilla de Araguaia.

La mayoría de hombres (excepto dos de ellos que tenían edad un poco avanzada y el pajé) fueron forzados a servir de guías, acompañar a los marehai en su búsqueda en la selva de los terroristas. "Ocuparam a aldeia dos índios Suruí, obrigando-os a servirem de guias, espalharam bases militares por toda a região", puntualizaría Campos Filho (2012: 151).

Los hombres que fueron forzados a ir junto a los militares eluden situaciones de malos tratos, hambre, privación de sueño, tensión constante. Aquellos que fueron cooptados, guardan dentro de su memoria, episodios marcantes que tienen que ver con el proceso de aniquilación de los guerrilleros o 'paulistas'. A más de los malos tratos a los que se vieron sometidos durante las jornadas de caza, los hombres Aikewara fueron testigos forzados de escenas de tortura a campesinos locales y de la decapitación de algunos de los ex guerrilleros.

\section{b). Quienes se quedaron}

A partir de 1972, una base militar de operaciones fue instalada junto al río Gameleira (limítrofe de la actual Tierra Indígena Sororó), en la localidad denominada São Raimundo, campamento militar localizado apenas a $100 \mathrm{~m}$. de la aldea.

Mientras el primer grupo mencionado fue forzado a acompañar a los militares en la selva, el resto de la población se vería confinada a quedar prácticamente presa al interior de la aldea, con restricciones de movilidad, acceso a alimentos, dinamización de prácticas culturales y sociales.

El pajé (líder espiritual), las mujeres y los niños son quienes re- construyen de este modo otra memoria, que retrotrae escenas de temor frente a un estado de excepción que fue instalado, así como por la preocupación latente por sus esposos/padres cooptados como 'guías'. Es significativo al respecto, que incluso la fiesta ritual de los karuara $^{22}$ que los Aikewara habían planificado tuvo que ser cancelada a causa de la intromisión militar; su cotidianidad se vio trastocada en diferentes niveles y en distintas dimensiones.

La limitación impuesta por los militares al interior de la aldea ocasionó inevitablemente una progresiva carencia de alimentos - que no podían ser sembrados ni cosechados-. Las mujeres comentan que lograron sobrevivir en base al consumo principal de papas y bananas: Disparos, ametralladoras, sonidos estridentes, tiroteos, explosión de granadas pasaron a formar parte de su cotidianidad, así como incendios más frecuentes. La propia convivencia con los militares en su tierra ya permite dimensionar los efectos de ello para las mujeres que quedaron 'presas'. Se tiene conocimiento de muerte de niños prematuros y de abortos ocurridos en medio de este panorama de tensión constante.

22 Siguiendo a Laraia: “dados obtidos em nossas pesquisas entre os surui e os akuawa-asurini, realizadas entre 1961 e 1966, nos levou a considerar os karuara como um tipo especial de espíritos, diferente dos owera, espíritos que abandonam o corpo quando as pessoas dormem, permitindo a entrada do asonga, espíritos que provocam os sonhos. Os karuara são invocados pelos pai'é (xamãs) para curarem as doenças, mas quando descontrolados são causadores das mesmas e podem até mesmo provocar a morte" (Laraia, 2009: 6). La realización de este ritual requiere del abastecimiento de varios alimentos, principalmente de la mandioca. Espíritus de los pajés muertos, que pueden causar enfermedades, si no son realizadas las prácticas rituales necesarias. 


\section{Recordaciones de lo común}

De cierta manera los estudios de memoria colectiva nos conducen a aproximaciones analíticas sobre modos de definición de los grupos en sí, los cuales poseen formas particulares de experimentar y evocar el pasado. Estos 'conjuntos de recuerdos' nos permiten reflexionar acerca de la ilusoria dicotomía creada entre lo externo e interno, entre lo individual y colectivo, al reconocer que la producción social se procesa en el interior de los sujetos, fundiéndose así en estos 'conjuntos' tanto referencias históricas como fragmentos personales.

Esta memoria que he denominado de 'lo común', evoca también a la misma como un bien distribuido de forma desigual en el interior de una sociedad en función de diversas posiciones estructurales que son ocupadas por los actores, y con ellos, diversas formas de afectación y de narrativas.

Habiendo analizado las fuentes disponibles donde constan testimonios que se refieren a este tipo de memoria más común, me fue posible detectar que los elementos fundamentales que fueron reseñados como parte de este conjunto de recuerdos compartidos acerca del evento son: el primer momento de encuentro con los marehai, la intervención de la Funai, las amenazas territoriales y la construcción de la Operacional 2 (OP2).

Todos los Aikewara que fueron testigos partícipes de la intromisión militar en su aldea evocan reiterativamente las impresiones que tuvieron al ver posar grandes helicópteros, que hasta ese entonces eran completamente desconocidos por ellos. Y junto con estos nuevos artefactos llegaron asimismo nuevos tipos de 'kamarás' (no indios, blancos) y con ellos, nuevas terminologías como: terroristas, dictadura, guerrilla, democracia; palabras 'de blanco' que estaban vaciados de sentidos desde la concepción nativa.

Recordemos que su primer contacto fue solo en 1953, nueve años antes de que los primeros paulistas aparecieran en la región y después de ellos, la cadena de kamarás que fueron subsiguiéndose, primero para encontrarlos y aniquilarlos al ser considerados terroristas, y después para encontrar sus restos mortales, ya para en ese entonces de los llamados desaparecidos políticos.

Siendo que, para hacer frente al movimiento guerrillero en sí fue necesario un despliegue de otro tipo de kamarás: los kamará marehai o militares. Algunas veces en la aldea Sororó los oí referirse también como kamará punura ${ }^{23}$. Cuando les pregunté sobre el significado de ello, la traducción más cercana hacía alusión a un 'hombre blanco malo'.

Frente al panorama totalmente incierto, los Aikewara puntualizan que al inicio de la invasión militar a la aldea, ellos habrían apelado y demandado protección por parte de la Funai ${ }^{24}$, ocurriendo que debido a las re-estructuraciones que la propia institución estaba atravesando, ésta terminaría respondiendo más como agente mediador dado que en ese entonces la Funai tuvo el desafío de construir un nuevo modelo de indigenismo dentro de los principios de la doctrina de Seguridad Nacional.

Ferraz (1985) concluye que fue solamente con la instauración del puesto indígena de la Funai en 1972, los Aikewara pasarían a ser reclutados efectivamente por los servidores de la agencia gubernamental.

Por su parte, la construcción de un ramal de la Transamazónica, la Operacional 2 (OP2) -hoy, BR-153-, sería inaugurada por el Ejército nacional en 1972, a raíz de la eclosión de la Guerrilla de Araguaia. En su caso particular, la porción oriental del Área Indígena Sororó se vio rasgada en una extensión de $11 \mathrm{~km}$. Posterior a ello, en 1981 ésta fue ampliada principalmente a causa de los

23 Dentro de la cosmovisión Aikewara, 'punura' vendría a ser un término, una categoría que engloba todo aquello que es considerado podrido, descompuesto.

24 Recientes análisis (Campos, 2012, Freitas, 1999; Silveira, 2000) muestran que en el período instaurado desde el Golpe de 1964 hasta 1985, la política indigenista del país contempló casos de desplazamientos y recolocaciones forzadas de poblaciones indígenas y el impedimiento de libre tránsito de las mismas. El monopolio de la mediación instaurado con la figura de la Funai, permitió dar mayor alcance a las disposiciones del régimen en varios puestos indígenas, consiguiendo la liberación progresiva de tierras y territorios con miras productivas. 
intereses colocados en los recursos minerales de la región fundamentalmente; siendo asfaltada a inicios de la década de noventa.

Basándonos en la lectura de varios testimonios tomados en la aldea Sororó, se obtuvo que las personas que fueron parte de la 'época de la guerra' recuerdan que los militares recurrían a uso de discursos para infundir temor sobre supuestas intenciones de los guerrilleros -ahora terroristas- sobre su territorio. Reconociendo que los argumentos militares usados en la aldea a veces también adquirieron tonalidades de amenaza de pérdida territorial.

De tal manera, es posible detectar una simbiosis de tres memorias surgidas en base al episodio de irrupción militar en la tierra indígena: de los hombres que fueron 'secuestrados' por los militares para las misiones de búsqueda y rastreo de guerrilleros; de las personas que quedaron presas en la aldea bajo dinámicas de un estado de sitio; $y$, una memoria que de cierta manera es más común a todos, por haber sido vivenciada por cada uno de ellos previo al distanciamiento forzado. No obstante, esta manera de dividir los recuerdos en base al evento de Araguaia responde a una interpretación de lo que podría ser la correlación de un tipo de memoria de alcance más común, y otros dos 'grupos' de memorias más especializadas surgidas al interior de la aldea que guardan coherencia con la noción de contextos mnemónicos.

Habiendo reconocido que en base al evento represivo de la Guerrilla de Araguaia, hubo una especie de tripartición de memorias entre los Aikewara, resulta importante justificar el motivo de tal división entre los recuerdos de quienes fueron y quienes quedaron en la aldea.

\section{Después de 'Araguaia'}

Cuando se logró poner fin al movimiento guerrillero en Araguaia en 1974, el Ejército aparentemente se retiró de la zona, no obstante, después de la ocurrencia fáctica del conflicto armado-y que como se vio, colocó tanto a campesinos como a los Aikewara en medio de un 'fuego cruzado'- se desataba aún una 'segunda guerra' o como Peixoto $(2008,2011)$ lo ha denominado: la 'guerra que veio depois', para referirse al crecimiento de conflictos año a año en la región sur de Pará, posterior al evento de Araguaia.

Al final de 1974, buena parte de las tropas de las fuerzas oficiales aún permaneció en la región para "limpiarla" ${ }_{25}$ bajo la coordinación del Coronel de Aeronáutica, Pedro Corrêa Cabral. Y "o que não se sabe ainda é o destino dado aos corpos dos desaparecidos do Araguaia... uma questão aberta que mantém vivo o episódio" (Fonteles, 2014: 7). El destino de los cuerpos de los desaparecidos ${ }^{26}$ políticos es un punto crucial para entender la vinculación directa que tiene la 'historia' de Araguaia con la 'historia' Aikewara, dado que según la documentación estudiada, se conoce que al final de la tercera campaña militar, los agentes de información e inteligencia presentes en la región indicaron los puntos donde estaban enterrados los cuerpos, desde donde eran embarcados en helicóptero para ser trasladados a la Sierra de las Andorinhas ${ }^{27}$.

25 Se refiere a las llamadas operaciones limpieza' dirigidas por el ejército nacional para eliminar cualquier vestigio en Araguaia post Guerrilla de Araguaia.

26 Pese a que en el presente trabajo no se realizan profundizaciones en torno a las implicaciones y alcances analíticas de la categoría de desaparecido, se quiere por lo menos llamar la atención de que esta categoría adquirió un trasfondo semántico más definido a partir de los movimientos sociales post dictadura argentina.

Por su parte, el 10 de diciembre de 2014, la Comisión Nacional de la Verdad en Brasil hizo la entrega oficial del Informe final, en el que se reconoce la cifra de 434 personas que fueron muertas o desaparecidas durante el régimen, de los cuales, cerca de 61 corresponden a los guerrilleros de Araguaia (sumando a ello, los campesinos que se habían alistado a la Fuerzas Revolucionarias y otros moradores que fueron tratados como terroristas por su supuesta vinculación o apoyo a los militantes). Cfr. CNV. Relatório Final, Vol. 1. Dez. 2014. Pp 715

27 Esta elevación natural tiene una importancia radical entre los Aikewara, puesto que es considerada como la morada de los espíritus y hoy en día forma parte del territorio 'perdido' por los Aikewara. 


\section{Al encuentro con los desaparecidos}

La búsqueda por los cuerpos de Araguaia ha conformado una especie de red que articula a los familiares, tiempo después al Estado, -compelido éste por obligaciones internacionales ${ }^{28}$ en el campo de los derechos humanos-, incluyéndose en esta red también a los Suruí Aikewara, quienes han pasado a formar parte de este proceso de otorgación de responsabilidades de acuerdo a su supuesto conocimiento de los posibles lugares de entierro de los cuerpos, como por la importancia de su territorio para el tema de búsqueda de restos mortales.

Los Aikewara fueron visitados por personal del Ejército, por familiares, por miembros de equipos interministeriales como el ex Grupo de Trabajo Tocantins, ahora convertido en Grupo de Trabajo Araguaia; y en las últimas temporadas también por representantes de la CNV (GT Araguaia). Recuerdan como algunos militares (kamará marehai) visitaban después de terminada la 'guerra' algunos lugares que están dentro o en los alrededores de su territorio. Este pueblo fue testigo entonces de este proceso de enterramiento y des-enterramiento (de cuerpos o restos óseos, según el caso) y de alguna manera asumieron una parte de la responsabilidad sobre los desaparecidos políticos.

Conforme fueron pasando los años y los esfuerzos por encontrar los restos de los ex militantes se dificultan más, algunas materias periodísticas comenzaron a aparecer haciendo mención a una supuesta colaboración de los Aikewara en el evento de Araguaia. En algunos de estos reportajes, los miembros del grupo indígena son descritos como los 'cortadores de cabeza' de los ex guerrilleros, consiguiendo con ello generar en el espacio de discurso público más elementos para culpabilizar a los Aikewara.

El día en que las evidencias escritas de lo que estaba siendo dicho sobre ellos llegó a la propia aldea fue un momento de radical importancia puesto que a partir de allí es que principalmente las nuevas generaciones se han empoderado y abanderado procesos para re contar su versión de los hechos, dándose paso a un ejercicio narrativo con dinámica propia.

\section{La cuarta memoria: re-significación generacional}

Así como en el primer capítulo abordamos las tres memorias Aikewara sobre el evento, éste es el momento de referirnos a una cuarta memoria, que guarda estrecha relación con los procesos más actuales desencadenados en la aldea en torno a los modos de narrar y re-significar el relato, movimientos liderados principalmente por las nuevas generaciones en la aldea.

En los discursos dados por algunos jóvenes Aikewara al respecto de la guerra de Araguaia, el elemento rescatado es la conexión de su generación con la historia mediante los relatos proferidos por sus abuelos, padres, refiriéndose que ahora entienden por qué ellos contaban. "No meio de tantas lindas histórias, que aprendemos ouvindo para poder repassar adiante de geração em geração para que nunca acabem ou morram, não era dessa maneira triste e feia que nós gostaríamos de fazer parte da história do nosso país...29. Esta es una declaración importante puesto que da cuenta del proceso paralelo que los más jóvenes de la aldea llevan adelante: por un lado, es una reivindicación por el modo de narrar de sus antecesores y de sus historias que los conectan intergeneracionalmente; y por otro lado, de las distorsiones de información, que desde su parecer se ha hecho en el espacio de la 'controversia pública' sobre su pueblo y su relación con el evento de represión de la guerrilla de Araguaia.

28 La atribución de responsabilidad que el Estado brasilero asume frente a la identificación de los cuerpos de los desaparecidos de Araguaia extrapoló la dimensión de la justicia local, pasando a adquirir una connotación de interés internacional, regido por normativas específicas sobre los derechos humanos. La condenación al Estado por la CIDH en el caso conocido: "Gomes Lund e outros (Guerrilha do Araguaia) vs. Brasil" (ADPF 153/2008, julgada pelo Supremo Tribunal Federal). en junio de 1995, por medio de la organización Human Rights Watch y del Centro pela Justiça e o Direito Internacional (CEJIL), los familiares enviaron una petición a la Corte Interamericana de Derechos Humanos (CIDH) de la Organización de los Estados Americanos (OEA).

29 Fragmento de la carta del pueblo Aikewara entregada a la Comisión Nacional de la Verdad: "O que nós esperamos", leída en la CNV, 13 mayo 2014. 
Sobre la reivindicación del modo de transmisión y construcción de una narrativa, amerita valorar la manera particular conforme la cual los Aikewara aseveran dinamizar internamente los relatos, dotando de amplio valor a la palabra de los más antiguos y en el caso de la historia de Araguaia, de aquellos que participaron directamente.

En ese sentido es que en base a un proceso mediado de reconstrucción de la narrativa comenzó a evidenciar vitalidad y demostrar que el supuesto ethos del silencio estaba disipándose. Aprovechando las coincidencias ${ }^{30} \mathrm{e}$ incidencias surgidas en el camino, dos profesores de la aldea Sororó junto a la antropóloga Iara Ferraz y Orlando Calheiros llevaron a cabo la realización y revisión de una fase de levantamiento de testimonios, en base a los cuales se generaron como productos: un texto y pruebas testimoniales audiovisuales.

El texto resultante titulado "O tempo da guerra": os Aikewara e a guerrilha do Araguaia" (2014, en imprenta) fue asumido desde su construcción como una suerte de narrativa reivindicatoria que era concebido como la 'historia verdadera' desde la perspectiva nativa. Y fue justamente con esta versión mediada con la que un grupo de indígenas de la aldea Sororó llegaron hasta las instancias de la CNV en Brasilia y a la Comisión de Amnistía posteriormente, en donde pasarían a ser el primer pueblo indígena en Brasil a ser reconocido como aministiado político por las violaciones vividas durante régimen militar.

\section{Sujetos de derechos: entre lo individual y lo coletivo}

El 19 de septiembre de 2014, la noticia de que 14 indígenas del pueblo Aikewara, o llamados de 'Suruí de Pará' fueron reconocidos como amistiados políticos conforme las directrices actuales que rigen la Comisión de Amnistía en Brasil, tuvo un importante impacto y difusión en medios de diferentes redes virtuales al haberse registrado como el primer caso de pedido de disculpas formales del Estado a una población indígena a nivel nacional a causa de las violaciones del período de dictadura civil militar.

En base al análisis llevado a cabo sobre las sesiones públicas en las que los Aikewara aportaron con sus testimonios a modo de microetnografías ${ }^{31}$ al interior de instituciones sociales encargadas de la gestión de políticas de memoria en Brasil sobre este caso concreto fue muy interesante realmente ver de cierta forma a dónde nos conducía esta vez el trayecto indomable que la memoria iba delineando.

Durante el tiempo que pude compartir junto a los Aikewara, tanto dentro como fuera de la aldea, un elemento que fue una de las constantes en toda la trayectoria que fue marcándose era la preocupación por la recuperación territorial, fue desde sus inicios de alguna forma el eje centrifugador de esta problemática. Y fue durante la sesión en la Comisión de Amnistía donde finalmente el eje se sobre expuso.

Entre varias de las exigencias y procesos burocráticos y legales que los Aikewara atravesaron antes de llegar a ese día altamente simbólico por las implicaciones que acarrea siempre estuvo implícita y latente una insistencia por individualizar el dolor, el relato, fragmentar la experiencia para poder compensarla (o al menos con esa ilusión).

Sin embargo, la lucha de los Aikewara ha sido desde antes del aparecimiento y aniquilamiento de la guerrilla y despúes de ella enfocada a la defensa y recuperación territorial. De tal

30 Se habla de coincidencias dado que de cierta manera el informe Aikewara pudo surgir a causa de que desde la Comisión de Amnistía se pidió a los antropólogos de confianza (escogidos por los propios Aikewara) para que se levantaran dichas pruebas testimoniales con el objeto de complementar unos pedidos de amnistía de 12 casos de Aikewara, hecho que fue negociado a cambio de mayor colaboración de los indígenas durante el proceso de búsqueda de los restos de los desaparecidos.

Coincidencia también porque de la CNV también hubo un estímulo para que el informe sea levantado, proporcionando becas del PNUD para los dos profesores Aikewara que formaron parte del proceso de construcción de la narrativa local en base al evento militar en Araguaia.

31 Para más detalle de dichas microetnografías en espacios públicos de negociación y construcción política de la memoria en la que participaron los Aikewara remitirse a la tesis completa. 
forma, vemos conjugarse casi fluidamente a la memoria y al territorio, ambas como fuerzas recíprocamente complementarias.

En el Decreto 88.648 (publicado no DOU en 31 de agosto de $1983^{32}$ ) cuenta la homologación de 26.258 hectáreas, y para el 25 de enero de 2012, el Diario Oficial da União publicó un informe sobre la identificación y delimitación de la tierra indígena 'TuwaApekuokawera', que revela oficialmente las distintas fases de pérdida progresiva del territorio durante el régimen dictatorial y después en la llamada Nueva República.

Frente a ello, durante sesiones públicas los Aikewara se han valido para lanzar una especie de provocación al Estado, al solicitar que la compensación estipulada a modo de reconocimiento de amnistía otorgada sea de carácter colectivo y expresada en la delimitación y aceleramiento del proceso de nueva delimitación del territorio, el mismo que quedaría fragmentado también despúes de la existencia de la actual autopista BR- 153.

Lo que salta a la vista, es la relevancia radical que tiene la deliberación colectiva como forma propia de resolver conflictos y el deber ser propuesto a modo de un concepto que revela una conexión interna e inseparable entre lo "consistente y lo no-consistente" con la realidad local (Íbid, 1998). En la aldea Sororó se reivindica constantemente que no fue individual el daño causado; al contrario, es compartido tanto por los que acompañaron forzadamente a los militares en su caza, como por los que quedaron "presos" en sus casas y actualmente por las recientes generaciones: es un trauma que los abarca a todos ${ }^{33}$.

Así, una reparación individual no se engendraría en una noción de pueblo Aikewara, en la medida en que se entienden como productos y productores de sí mismos y de sus redes de interacción. Si todo sujeto, acción o cosa, como nos dice Marylin Strathern (1988) no existe fuera de la relación que los produce, la demanda de reparación agenciada por el reconocimiento de sus territorios, afectados por las acciones de la dictadura militar en diferentes aspectos, podría, desde sus regímenes de conocimiento y desde su sociopolítica, garantizar la sustentación de su universo social.

Evaluado todo el proceso antes descrito se podría decir que los Aikewara pasaron también al mismo tiempo por un tránsito de ser reconocidos como víctimas, pasando luego a la categoría de sujetos de derechos. El hecho de ser reconocido como el primer pueblo indígena que ha recibido disculpas formales por parte del Estado brasilero a causa de las violaciones a las que se vieron sometidos por parte del propio Estado, juntamente con el reconocimiento económico -individualizado y debidamente justificado según cada caso particular-, abre pautas para pensar tal vez más integralmente las relaciones entre pueblos, mundos indígenas y concepciones y categorías de perdón, justicia, reparación.

Imagino que estudios posteriores podrán abrir aún más la discusión después de que sea posible comenzar a evaluar los efectos de la entrada de aquellas compensaciones al interior de la aldea en términos por ejemplo de posible generación de nuevos códigos de diferenciación.

\section{Conclusiones}

Me gustaría concluir este artículo que resultó realmente complejo de sistematizar dada la cantidad de información recabada (la mayoría de ella aún temporalmente depositada en mis recuerdos), colocando que la complejidad que envuelve esta temática es tal, que por un lado nos

32 Se agrega que: "Em 1982 a FUNAI recebeu manifestações de que a área demarcada era muito inferior àquela necessária à reprodução física e cultural dos Suruí/Aikewar. O departamento fundiário da FUNAI exarou documentos reconhecendo que a área demarcada realmente não atendia as necessidades do povo. No entanto, apesar disso, em 1983 a área foi homologada e registrada..." (DOU/Fundação Nacional do Índio, 24/01/2012).

33 Asimismo como en el Laudo (2008) se evoca el hecho de que cuando los tres Suruí que habían sido juzgados como terroristas, el reclamo general había estado direccionado a posicionar que la responsabilidad de lo allí ocurrido -cierre de la carretera, sanción mediante uso de la paxiba- era de todos y no sólo de los tres que aparecían en el "Boletim de Ocorrência da Delegacia da Policia Civil em São Geraldo do Araguaia" (Sob N. 2003.000110 por la Delegada Cláudia Pimentel R. en donde se encuadró el hecho como robo simple y tentativa de homicidio) (Beltrão, 2008: 220). 
devela el pedido aparentemente consensuado y aprobado colectivamente en la aldea Sororó para un pedido de reparación en su territorio, mientras al mismo tiempo estén surgiendo actualmente nuevas propuestas de fragmentación de nuevas aldeas entre algunas familias. La memoria del territorio conduce a que éste sea ocupado de manera más extensiva, conforme sus narrativas también van ganando espacio.

Considero que el paso de un recuerdo individual para una memoria colectiva, que construye y pacta lo que será transmitido en los espacios públicos, en la medida en que la narrativa en proceso está asociada a una 'lucha por los derechos'.

Por tal motivo, en el tercer capítulo nos centramos en la cuestión del manejo y construcción de la imagen pública de los Aikewara, en donde fueron algunas veces estigmatizados. Considerando para ello, el impacto del acceso a las narrativas foráneas de manera escrita, las mismas que daban cuenta de imaginarios construidos sobre los Aikewara y su participación en el evento, colocándolos muchas veces en dicotomías rígidas como 'víctimas/victimarios'.

Como se vio, frente a ello, una cuarta memoria surge en el momento en que principalmente las nuevas generaciones han pasado a ser portavoces de los relatos que sus abuelos o padres contaban para ellos en la intimidad de la familia, de la aldea. De tal suerte, la narrativa ha incorporado recuerdos individuales y colectivos además de las más recientes re-elaboraciones discursivas a cargo de jóvenes de la aldea que han tenido otro tipo de contacto y comunicación con los kamará en especial, a causa de escolarización y por contacto con más actores sociales que conforman la red en torno al evento de Araguaia.

Pienso que el seguimiento a la trayectoria de la(s) memoria(s) Aikewara desde la guerra de Araguaia hasta recientes eventos de amnistía política nos permitió dar cuenta de que el tema recurrente a lo largo de esta narrativa fue siempre el territorio. Éste como hilo conductor en torno al cual se activan distintos dispositivos y gramáticas de la memoria individual y colectiva de un pueblo que está en constante re formulación (nuevas aldeas están siendo formadas, expandiéndose en el territorio actual).

Durante toda la investigación los doblamientos y des-doblamientos del silencio otorgaron pautas para pensar la trayectoria de la(s) memoria(s) Aikewara. El uso estratégico del silencio como modo de resistencia es algo que invariablemente es colocado como fondo de una serie de agenciamientos políticos posteriores que permiten visualizar el cruce entre memoria y territorio, entre memoria y narrativa.

El pasado no visto más como un objeto que puede ser sencillamente accequible, sino más bien, asimilado como un trabajo en donde las identidades son colocadas en juego y disputas. "Se é verdade que no campo da memória ocorre uma seleção dos momentos do passado e não o seu total arquivamento, ou seja a memória só existe ao lado do esquecimento..." (Seligmann, 2000: 83- 84).

Me parece que en la base de todo el trayecto nmenónico analizado, sentido, estuvo siempre presente la provocación, la negociación o la batalla entre categorías, muchas de las cuales son re-significadas y re-actualizadas evidenciando cambiantes procesos de dotación de sentidos. Tal vez sea por ello que en las manifestaciones o demandas públicas que los Aikewara realizan de vez en cuando para reclamar principalmente asuntos relativos a efectos de la autopista en su territorio, se les escuche valerse de comparaciones de su lucha con la de los guerrilleros, los comunistas... 


\section{Bibliografía}

Abrão, P.; Torelly, M. 2014, "Mutações do conceito de anistia na justiça de transição brasileira: a terceira fase da luta pela anistia", en: Piovesan, F.; Prado, I. (Coord.) Direitos humanos atual, Rio de Janeiro, Elsevier, pp. 112- 18 .

Arruti, J. M. P. A; Montero, Paula; Pompa, C. 2012. "Para uma Antropologia do político", en: Adrian Gurza Lavalle (org.). O horizonte da Política: Questões emergentes e agendas de pesquisa. São Paulo: UNESP, v. 1, pp. 145-184.

Bosi, E. 1987, Memória e Sociedade - Lembrança de velhos, São Paulo, T.A. Queiroz/ Edusp, 1987, pp. 66-67.

Calheiros, O. 2014, Aikewara, Esboços de uma sociocosmologia tupi-guarani, Tese de Doutorado em Antropologia Social, Rio de Janeiro, UFRJ [Orientador Prof. Eduardo Viveiros de Castro].

Das, V. 2007, Life and words: Violence and the descent into the ordinary, Berkeley: University of California Press.

1995, Critical Events. An Anthropological Perspective on Contemporary India, New Delhi, Oxford University Press.

De Souza, E. 2012, Situação Sociolinguística da língua Suruí do Tocantins, Trabalho de Conclusão de Curso apresentado para obtenção do grau de Licenciado em Letras, Belém: UEPA.

Dória, P.; Buarque, S.; Carelli, V.; Sautchuk, J. 1978, A Guerrilha do Araguaia. Col. História Imediata, São Paulo, Ed. Alfa-Omega.

Fernandes, N. 2013, Memória social e Guerrilha do Araguaia, Dissertação de Mestrado em Sociologia, Goiânia, UFG [Orientador Prof. Nildo Silva Viana].

Freitas, E. 1999, Índios- soldados. A GRIN e a tradição militar da política indigenista Brasileira, Tese de Doutorado em História Social, São Paulo, USP [Orientador Prof. Marcos A. da Silva].

2011, "A Guarda Rural Indígena- GRIN. Aspectos da militarização da política indigenista no Brasil”, trabalho apresentado no Simpósio Temático 'Os índios e o Atlântico', XXVI Simpósio Nacional de História da ANPUH, São Paulo, 17 a 22 julho.

Ferraz, I. 1985, "Suruí/Aikewara do Posto Indígena Sororó: no rastro do território tradicional” (Relatório cf. Portaria FUNAI 1981/E de 07.02.1985). Brasília: mimeo.

2013, Memória e história no baixo Araguaia segundo os Aikewara da Terra Indígena Sororó. Subsídio para a Comissão Especial sobre Mortos e Desaparecidos Políticos da Secretaria de Direitos Humanos da Presidência da República. Brasília.

Ferraz, I.; Calheiros, O; Suruí, T.; Suruí, Y. (Org.). “O tempo da guerra”: os Aikewara e a guerrilha do Araguaia. Relatório apresentado à Comissão Nacional da Verdade, Brasília, maio de 2014, prelo.

Fonteles, P. 2013, Araguaianas. As histórias que não podem ser esquecidas, São Paulo, Fundação Maurício Grabois e Ed. Anita Garibaldi.

Gorender, J. 1987, O combate nas trevas. A esquerda brasileira: das ilusões perdidas à luta armada, São Paulo, Serie Temas, Vol. 3 Brasil Contemporâneo.

Laraia, R.; Da Matta, R. 1978, Índios e castanheiros. A empresa extrativa e os índios no Médio Tocantins, Rio de Janeiro, Paz e Terra, 2.a ed. (Coleção Estudos Brasileiros, v. 35).

Nossa. L. 2012, Mata! O Major Curió e as guerrilhas no Araguaia, São Paulo: Companhia das Letras, 1 ed.

Peixoto, R. 2011, “Memória Social da Guerrilha do Araguaia e da guerra que veio depois”. Belém: Bol, Museu Paraense Emílio Goeldi, Col. Ciências Humanas, v.6, n.3, pp. 479- 499.

2014. "Índios e camponeses: antes, durante e depois da Guerrilha do Araguaia". Revista Territórios e Fronteiras. Cuiabá, vol.7, n. 1.

Ricardo, C. 2013, (ed.). Povos indígenas no Brasil, Vol.8: Sudeste do Pará/Tocantins, 100-125, São Paulo, CEDI.

Relatório. 2014, Grupo Tortura Nunca Mais, SP. 36 pp.

Ross, F. 2006, "La elaboración de una Memoria Nacional: la Comisión de Verdad y Reconciliación de Sudáfrica", en: Cuadernos de Antropología Social $N^{\circ}$ 24: 51- 68.

Seligmann-Silva, M. 2008a, "Narrar o trauma", en: UMBACH, R. Memórias da repressão, UFSM, PPGL, pp. 73- 92.

Strathern, M. 1988, The Gender of the Gift: Problems with Women and Problems with Society in Melanesia, University of California Press. 
Silveira, J. G. 2000, A ordem a se preservar: a gestão dos índios e o reformatório agrícola indígena Krenak, Dissertação de Mestrado, Rio de Janeiro, UFRJ.

Souza Lima, A. 1995, Um grande cerco de paz: poder tutelar, indianidade e formação do Estado no Brasil, Petrópolis (RJ), Ed. Vozes.

Vieira, E. Diário PA - Executivo de 24/02/2012 (5370784889438208). Resumen del Relatorio circunstanciado de identificación y delimitación de la tierra indígena Tuwa Apekuokawera (Processo FUNAI/BSB/2047/2004). En: http://www.radaroficial.com.br/d/5370784889438208.

1996. "Novos subsídios para a revisão de limites (acréscimo) da Terra Indígena Sororó /PA". Departamento de Identificação e Delimitação DID/DAF/FUNAI. Brasília: mimeo. 